\title{
Navigated Pin-Point Approach to Osteoid Osteoma Adjacent to the Facet Joint of Spine
}

\author{
Kanji Mori ${ }^{1}$, Masashi $\mathrm{Neo}^{2}$, Mitsuru Takemoto ${ }^{3}$, Kazuya Nishizawa $^{1}$, Shinji Imai ${ }^{1}$ \\ ${ }^{1}$ Department of Orthopaedic Surgery, Shiga University of Medical Sciencea, Otsu, Japan \\ ${ }^{2}$ Department of Orthopedic Surgery, Osaka Medical College, Osaka, Japan \\ ${ }^{3}$ Department of Orthopaedic Surgery, Graduate School of Medicine, Kyoto University, Kyoto, Japan
}

\begin{abstract}
Osteoid osteoma (00) is a benign osteoblastic tumor. Its curative treatment is complete removal of the nidus, where intraoperative localization of the nidus governs clinical results. However, treatment can be difficult since the lesion is often invisible over the bony surface. Accordingly, establishment of an ideal less invasive surgical strategy for spinal 00 remains yet unsettled. We illustrate the efficacy of a computed tomography (CT)-based navigation system in excising 00 located adjacent to the facet joint of spine. In our 2 cases, complete and pin-point removal of the nidus located close to the facet joint was successfully achieved, without excessive removal of the bone potentially leading to spinal instability and possible damage of nearby neurovascular structures. We advocate a less invasive approach to spinal 00, particularly in an environment with an available CT-based navigation system.
\end{abstract}

Keywords: Osteoid osteoma; Spine; Computed tomography-based computer navigation system; Less invasive

\section{Introduction}

Osteoid osteoma (OO) is a benign bone tumor originally described by Jaffe [1] in 1935. OO predominantly localizes in the long bones of extremities, while its occurrence in the spine remains as low as $10 \%$ to $20 \%$ [2]. Its curative treatment is complete removal of the nidus. Intraoperative localization of the nidus governs clinical results $[3,4]$. However, treatment can be difficult since the lesion is often invisible over the bony surface $[3,5]$. Furthermore, we must remember that spinal fusion becomes necessary when spinal stability is compromised by a wide resection of the affected vertebrae.

In recent years, a computer-assisted navigation system has become increasingly popular among spinal surgeons. Many reports emphasize that it increases the accuracy of implantation [6] and bone resection in cases with ossification of the spinal ligaments $[7,8]$ and bone tumors [5,9-12] by providing real-time information.

In the present report, we describe 2 cases with OO adjacent to the facet joint of spine. Both cases were successfully treated with a pin-point excision of the nidus without compromising spinal stability or damaging neurovascular structures using a computed tomography (CT)-based navigation system.

\section{Technical Note}

From 2008 to 2011, 2 cases of OO adjacent to the facet joint of spine were treated with CT-based navigation surgery in our institutes. Written informed consent was obtained from the patient for publication of this report and

Received Mar 17, 2015; Revised Apr 9, 2015; Accepted Apr 11, 2015

Corresponding author: Kanji Mori

Department of Orthopaedic Surgery, Shiga University of Medical Science, Tsukinowa-cho, Seta, Otsu, Shiga 520-2192, Japan

Tel: +81-77-548-2252, Fax: +81-77-548-2254, E-mail: kanchi@belle.shiga-med.ac.jp 
any accompanying images.

\section{Navigation-assisted surgery}

The navigation systems used were StealthStation TRIA (Medtronic Sofamor Danek, Memphis, TN, USA) for case 1 and VectorVision (Spine ver. 5.6, Brain Lab, Feldkirchen, Germany) for case 2. Both systems are commerciallyavailable CT-based navigation systems. The basic data used for navigation consist of preoperative CT data (slice thickness, $1 \mathrm{~mm}$ ), which are transferred and recorded on the system computer and reconstructed into three-dimensional (3D) images.

The registration procedures of point merging and surface merging produces $3 \mathrm{D}$ spinal images on the monitor that are identical to the actual appearance of the spine in the operative field. The accuracy of the images is verified by placing a tool navigator on various parts of the exposed area.

\section{Case 1}

A 40-year-old man suffered from localized left lower back pain with a 3-year history. The pain deteriorated gradually, particularly during night time. His sleep was constantly disturbed by unremitting pain during the previous year. He visited an orthopaedic clinic where OO of L2 was identified by CT (Fig. 1A) and magnetic resonance (MR) imaging. Nidus was localized in the subchondral region of the left superior articular process of L2 (Fig. 1A). Treatment with non-steroidal anti-inflammatory drug (NSAID) was effective, however, this treatment was halted due to a drug-induced gastric ulcer.

Subsequently, he was introduced to us for surgical treat- ment. Physical examination revealed only a localized tenderness over the left L1/2 facet joint without neurological compromise. After paramedial skin incision, the left L1/2 facet was exposed by splitting multifundus and longissimus muscles. Reference frame was placed to the tip of the L2 spinous process following minimum exposure. Registration was performed by tracing the spinous process, left transverse process, left lamina and left superior articular process of L2. Macroscopically, we could not localize the nidus over the bony surface. Following navigation guidance, a precise localization of the nidus became possible. En-block extirpation was performed from the extra-articular aspect of the superior articular process of L2 without exposing its articular surface. The pin-point approach to the nidus enabled maximum preservation of capsular attachment to the facet joint. Histological examination revealed that the resected specimen was compatible with OO.

Postoperative CT taken immediately after the operation revealed complete and pin-point removal of the nidus (Fig. 1B). At the time of the latest follow-up, 2.5 years after surgery, the patient was free of symptoms, and there was no clinical or radiologic evidence for instability and recurrence of the tumor (Fig. 2). Bone defect of left superior articular process of L2 was completely remodeled postoperatively (Fig. 1C).

\section{Case 2}

A 16-year-old man complained of right neck pain. A plain X-ray of the cervical spine at the initial consultation showed a slight torticollis. MR imaging revealed an inflammation/edema pattern at the right side of $\mathrm{C} 2$ lamina (Fig. 3) with the diagnosis of bone bruise. He enjoyed
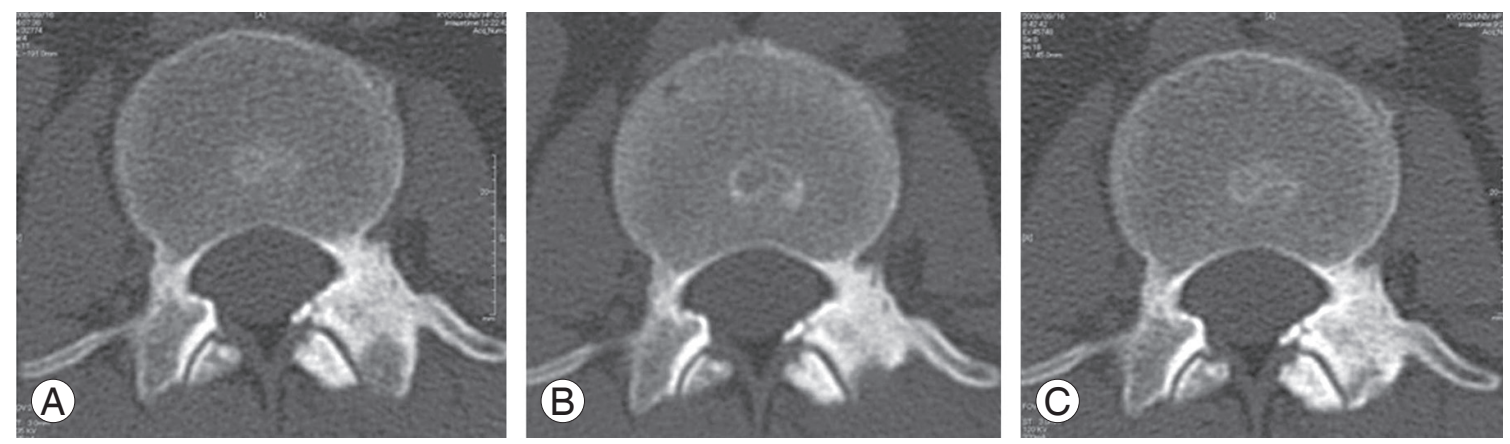

Fig. 1. Case 1. Computed tomography images. Preoperative (A), Postoperative (B) and one-year follow-up (C) computed tomography images. Pin-point extirpation of the nidus without excessive bone resection (B) and its remodeling (C) was observed. No tumor recurrence was detected. 
playing Judo, though he reported no preceding severe trauma. NSAID was effective during an early stage of his illness, though pain aggravated gradually.

Six months after the onset of symptoms, he was referred to us for persistent neck pain. On physical examination, right rotation of the neck was noticeably limited with no neurological compromise. Second-look MR imaging demonstrated a persistent abnormality. Subsequent CT demonstrated a small round nidus at the base of right inferior articular process of C2 (Fig. 4A, B). Technetium bone scan showed a focal uptake at an area corresponding to the lesion detected by CT and MRI.

Under a tentative diagnosis of $\mathrm{OO}$ arising at $\mathrm{C} 2$, removal of the nidus was performed using a CT-based navigation system. To minimize possible navigational error caused by a change in intersegmental relationship after surgical positioning, we carefully positioned the patient to reproduce the alignment of $\mathrm{C} 2-3$ in preoperative $\mathrm{CT}$.
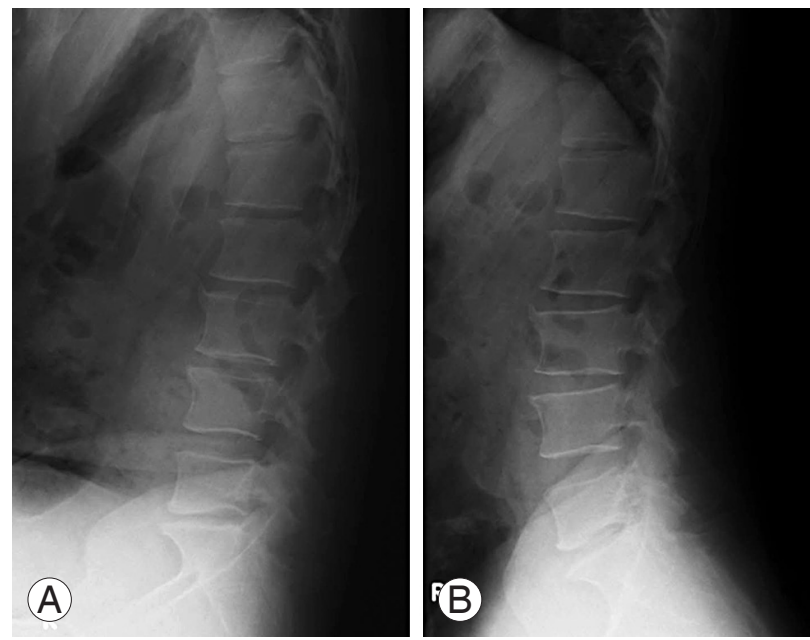

Fig. 2. Case 1. Postoperative plain X-ray of lumbar spine in flexion (A) and extension (B) positions at the latest follow-up, 2.5 years after surgery, showed no radiographic instability.
Right laminae of C2 and C3 were minimally exposed up to the lateral edge of facet joint. Extensor muscles attached to $\mathrm{C} 2$ and $\mathrm{C} 3$ were maximally preserved. The reference frame of the navigation system was set on the Kirchner wires inserted into the spinous process of $\mathrm{C} 2$ and C3. Registration was performed by tracing the inferior articular process of $\mathrm{C} 2$, the spinous process and the right laminae of $\mathrm{C} 2$ and $\mathrm{C} 3$.

Via navigation guidance, a trans-laminar tunnel reaching to the nidus was made using a high-speed drill. Then, complete removal of the nidus was achieved without sacrificing spinal stability (Fig. 5). The CT-based navigation system not only enabled pin-point removal of the nidus but also prevented possible damage of nearby critical structures (Fig. 5). Histopathological diagnosis of the resected lesion was that of OO (Fig. 6). Postoperative CT
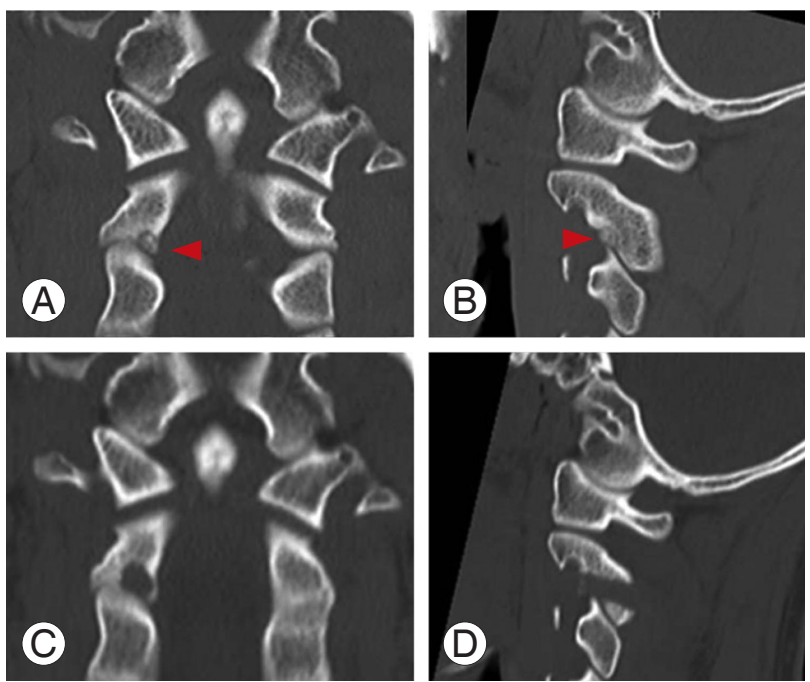

Fig. 4. Case 2. Preoperative coronal (A), sagittal (B), and reconstruction computed tomography (CT) images showing a nidus of C2 (red arrowheads). Postoperative coronal (C) and sagittal (D) reconstruction CT images revealed complete and pin-point removal of the nidus without excessive bone resection around the lesion.
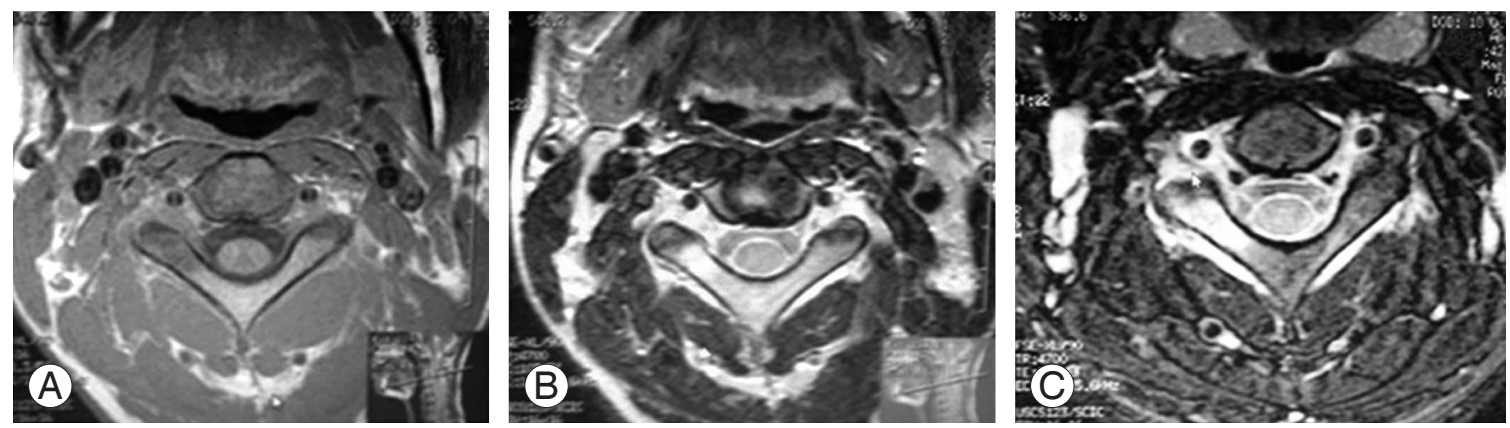

Fig. 3. Case 2. Preoperative magnetic resonance imaging of T1-weighted (A), T2-weighted (B) and short TI inversion recovery (C) images showing inflammatory/edema pattern intensity at the right side of the C2 lamina. 

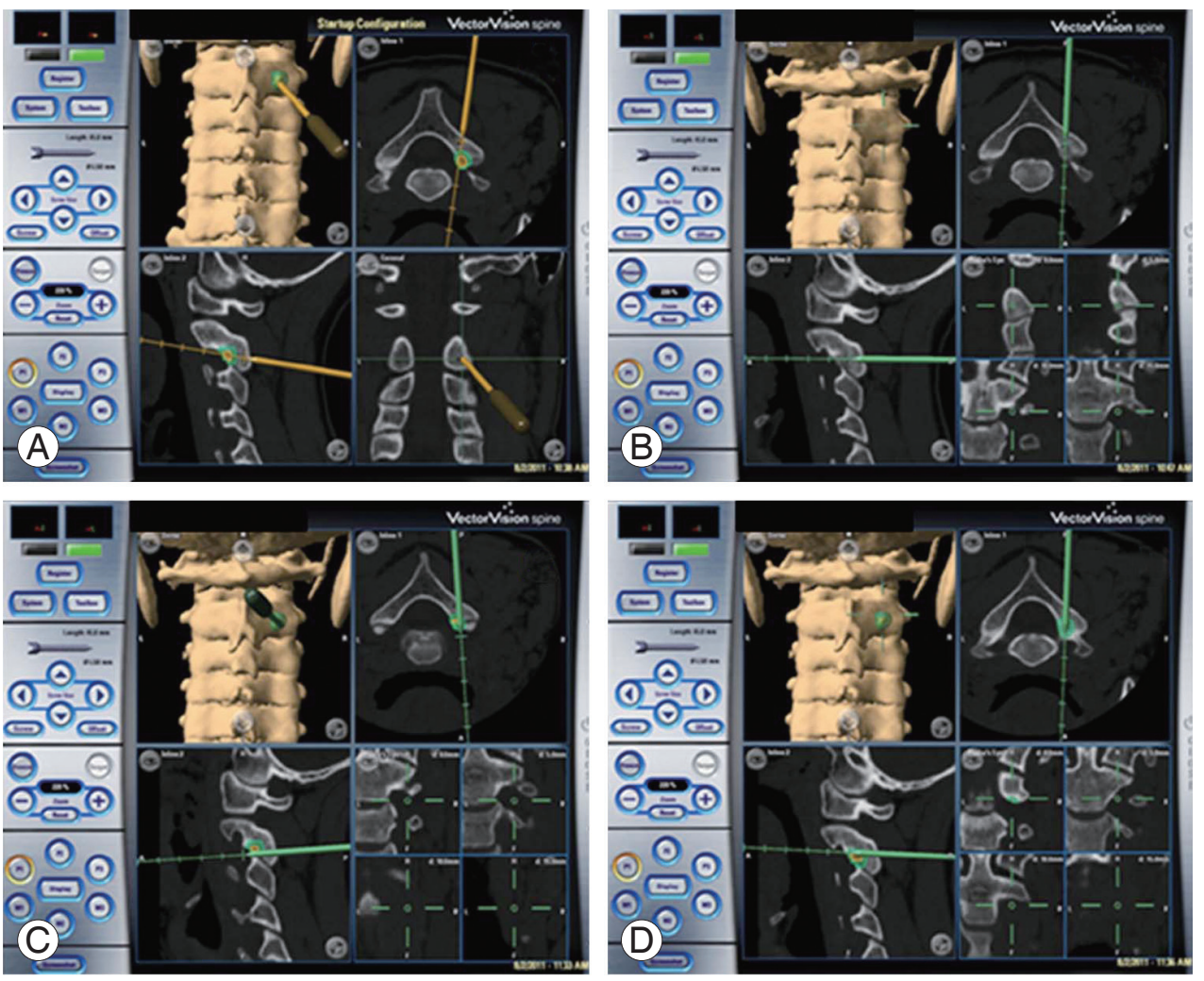

Fig. 5. Case 2. Intraoperative navigation monitoring. On the screen, three-dimensional, coronal, sagittal, and axial images in conjunction with the actual instrument tip image. Yellow (A) and Green (B-D) arrows indicate the position of the pointer in the operation field.

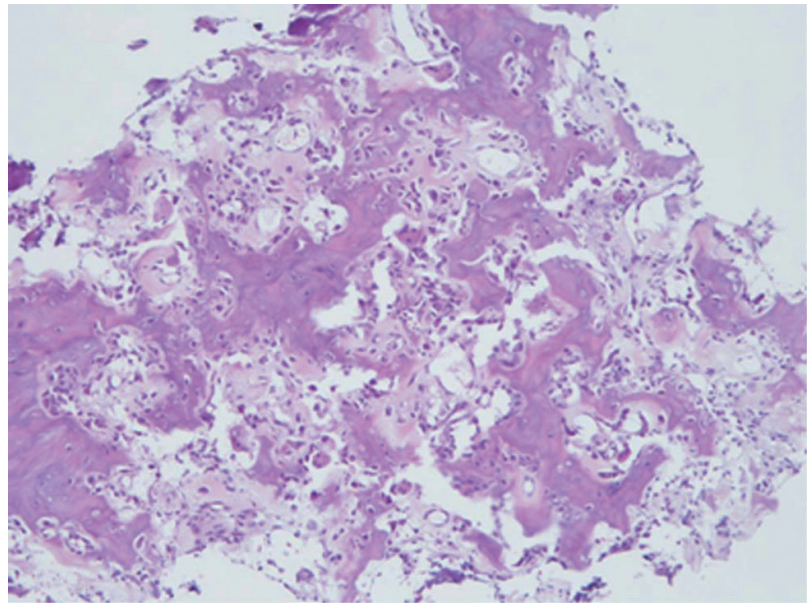

Fig. 6. Case 2. Histolopathological examination revealed that the extracted lesion showed an osteoid lined with plump osteoblasts and vascularized connective tissue $(H \& E, \times 100)$.

confirmed the complete removal of the nidus (Fig. 4C, D). At the time of the latest follow-up, 3.5 years after surgery, the patient was free of symptoms and there was no clinical or radiologic evidence for recurrence of the tumor (Fig. 7).

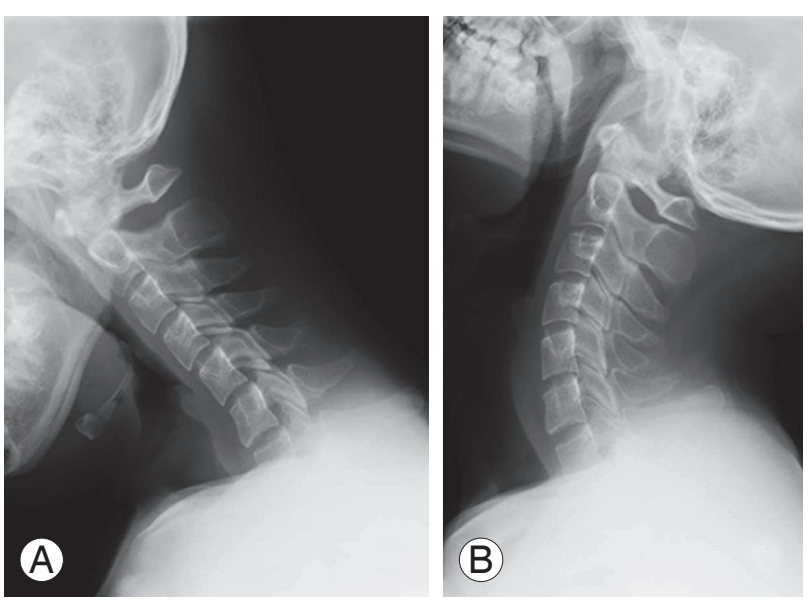

Fig. 7. Case 2. Postoperative plain X-ray of the cervical spine in flexion (A) and extension (B) positions at the latest follow-up, 3.5 years after surgery, showed no radiographic instability.

\section{Discussion}

Several therapeutic modalities to treat $\mathrm{OO}$ have been proposed to minimize surgical trauma. Such modalities 
include radiofrequency thermal coagulation $[2,13]$ or drill resection [14] under CT guidance. These techniques have been verified in the treatment of bone tumors of extremities. Spinal OO, however, may not allow a straightforward application of these techniques. Namely, application of these techniques in spinal OO carries a latent risk of damaging adjacent structures, such as the spinal cord, nerve root and vertebral artery [2,13-16]. Indeed, several authors have reported undesirable consequences resulting from damaging the neurovascular structures while performing these techniques on spinal tumors $[3,14,17]$.

Another critical drawback of thermal deactivation of the nidus with electrical coagulation resides in a lack of histological verification of the tumor [2,5]. Histological examination is recommended in order to confirm the diagnosis of the tumor [15]. Indeed, Sans et al. [15] reported that inconsistent histological diagnosis was made in 6 out of 38 patients with OO treated by CT-guided percutaneous drill resection.

In turn, the recent development of CT-based navigation surgery considerably contributed to spinal surgery. Van Royen et al. [11] reported 5 cases of spinal OO successfully treated with a CT-based navigation system combined with high-speed drill excision. They emphasized that the simultaneous use of $\gamma$ probe guidance with the CT-based navigation system greatly facilitated matching the spinal anatomy of the patient. However, this technique imposes radiologic exposure on the patient.

Rajasekaran et al. [5] reported use of the Iso-C 3D navigation system for the treatment of spinal $\mathrm{OO}$ in 4 cases. The authors emphasized advantages of this system over the CT-based navigation system: anatomical registration is unnecessary, multiple adjacent levels are registered simultaneously, and images for navigation are taken after intraoperative acquisition of optimal alignment. In addition, intraoperative excision is confirmed through reacquisition of data. However, the Iso-C 3D navigation may not provide as fine an image as the CT-based navigation system, particularly when localizing small lesions as in spinal OO [18].

Nagashima et al. [12] recently described a case with an $\mathrm{OO}$ of the $\mathrm{C} 2$ pedicle successfully treated by using a conventional CT-based navigation system. They used the CT-based navigation system simply to localize the bony lesion after an extensive exposure of the entire posterior compartment of the affected vertebra.

The CT-based navigation system does not only prevent damaging of adjacent neurovascular structures, but also allows pin-point, a minimally invasive approach reaching directly to the target lesion. Since excessive resection of bone and joint structures as well as detachment of spinal muscles greatly compromises spinal stability, we advocate a less invasive approach to spinal OO, particularly in an environment where a CT-based navigation system is available. However, we must keep in mind that computerassisted surgeries still carry latent errors. Iso-C 3D navigation and CT-based navigation have a mean deviation of 0.5 $\mathrm{mm}$ and $1.0 \mathrm{~mm}$, respectively [19].

\section{Conflict of Interest}

No potential conflict of interest relevant to this article was reported.

\section{References}

1. Jaffe HL. "osteoid-osteoma": a benign osteoblastic tumor composed of osteoid and atypical bone. Arch Surg 1935;31:709-28.

2. Hadjipavlou AG, Tzermiadianos MN, Kakavelakis KN, Lander P. Percutaneous core excision and radiofrequency thermo-coagulation for the ablation of osteoid osteoma of the spine. Eur Spine J 2009;18:34551.

3. Ozaki T, Liljenqvist U, Hillmann A, et al. Osteoid osteoma and osteoblastoma of the spine: experiences with 22 patients. Clin Orthop Relat Res 2002;(397):394-402.

4. Saccomanni B. Osteoid osteoma and osteoblastoma of the spine: a review of the literature. Curr Rev Musculoskelet Med 2009;2:65-7.

5. Rajasekaran S, Kamath V, Shetty AP. Intraoperative Iso-C three-dimensional navigation in excision of spinal osteoid osteomas. Spine (Phila Pa 1976) 2008;33:E25-9.

6. Rajasekaran S, Vidyadhara S, Ramesh P, Shetty AP. Randomized clinical study to compare the accuracy of navigated and non-navigated thoracic pedicle screws in deformity correction surgeries. Spine (Phila Pa 1976) 2007;32:E56-64.

7. Seichi A, Takeshita K, Kawaguchi H, Kawamura N, Higashikawa A, Nakamura K. Image-guided surgery for thoracic ossification of the posterior longitudinal ligament: technical note. J Neurosurg Spine 
2005;3:165-8.

8. Seichi A, Nakajima S, Takeshita K, et al. Image-guided resection for thoracic ossification of the ligamentum flavum. J Neurosurg 2003;99(1 Suppl):60-3.

9. Neo M, Asato R, Honda K, Kataoka K, Fujibayashi S, Nakamura T. Transmaxillary and transmandibular approach to a C1 chordoma. Spine (Phila Pa 1976) 2007;32:E236-9.

10. Neo M, Asato R, Fujibayashi S, Ito H, Takemoto M, Nakamura T. Navigated anterior approach to the upper cervical spine after occipitocervical fusion. Spine (Phila Pa 1976) 2009;34:E800-5.

11. Van Royen BJ, Baayen JC, Pijpers R, Noske DP, Schakenraad D, Wuisman PI. Osteoid osteoma of the spine: a novel technique using combined computerassisted and gamma probe-guided high-speed intralesional drill excision. Spine (Phila $\mathrm{Pa} 1976$ ) 2005;30:369-73.

12. Nagashima H, Nishi T, Yamane K, Tanida A. Case report: osteoid osteoma of the $\mathrm{C} 2$ pedicle: surgical technique using a navigation system. Clin Orthop Relat Res 2010;468:283-8.

13. Rosenthal DI, Hornicek FJ, Wolfe MW, Jennings LC, Gebhardt MC, Mankin HJ. Percutaneous radiofrequency coagulation of osteoid osteoma compared with operative treatment. J Bone Joint Surg Am 1998;80:815-21.

14. Assoun J, Railhac JJ, Bonnevialle P, et al. Osteoid osteoma: percutaneous resection with CT guidance. Radiology 1993;188:541-7.

15. Sans N, Galy-Fourcade D, Assoun J, et al. Osteoid osteoma: CT-guided percutaneous resection and follow-up in 38 patients. Radiology 1999;212:687-92.

16. Zambelli PY, Lechevallier J, Bracq H, Carlioz H. Osteoid osteoma or osteoblastoma of the cervical spine in relation to the vertebral artery. J Pediatr Orthop 1994;14:788-92.

17. Hadjipavlou AG, Lander PH, Marchesi D, Katonis PG, Gaitanis IN. Minimally invasive surgery for ablation of osteoid osteoma of the spine. Spine (Phila Pa 1976) 2003;28:E472-7.

18. Briem D, Linhart W, Lehmann W, et al. Computerassisted screw insertion into the first sacral vertebra using a three-dimensional image intensifier: results of a controlled experimental investigation. Eur Spine J 2006;15:757-63.

19. Geerling J, Gosling T, Gosling A, et al. Navigated pedicle screw placement: experimental comparison between CT- and 3D fluoroscopy-based techniques. Comput Aided Surg 2008;13:157-66. 